\title{
A Review of Intra vehicular Communication: Basics to Protocols
}

\author{
Pavan Arun Bhabat \\ Research Scholar, Electronics Engineering, \\ Rajarambapu Institute of Technology, Islampur Sangli 415414 (M.S) India \\ Mahesh S Kumbhar \\ Associate Professor, Electronics Engineering, \\ Rajarambapu Institute of Technology, Islampur Sangli 415414 (M.S) India
}

\begin{abstract}
In the present scenario, automotive electronics is at the pinnacle and much of the research is extensively carried out in European countries. Nowadays electronics had become part and parcel of the automobiles. Communication buses and its allied protocols are the integral part of this field. In order to catch hold of features exhibited by the modern and next generation protocols which are hot topics there is a need to relive the basics. This is a review paper which presents the know how's of the automotive electronics. This work would prove helpful for novice and young researchers facing the challenges to understand the electronics in automotive.
\end{abstract}

Keywords: CAN Controller Area Network, LIN, MOST media oriented system transport, LVDS low voltage differential signalling.

\section{INTRODUCTION}

The automotive electronics is a very rapidly changing field. Behind the protocols there are buses which perform their work hence introducing them becomes mandatory. A bus can be described as one or two wire media used for communication among nodes connected to it. A bus has the tabled number of types.

Table 1: Types of buses

\begin{tabular}{|l|l|}
\hline Type & Features \\
\hline $\begin{array}{l}\text { Single Ended } \\
\text { Unidirectional }\end{array}$ & $\begin{array}{l}\text { Used for one way communication } \\
\text { only and no acknowledgement can } \\
\text { be received by the sender }\end{array}$ \\
\hline $\begin{array}{l}\text { Single Ended } \\
\text { Bidirectional }\end{array}$ & $\begin{array}{l}\text { Used for two way but system cannot } \\
\text { tolerate large ground differences } \\
\text { among the connected nodes }\end{array}$ \\
\hline $\begin{array}{l}\text { Differential } \\
\text { Bidirectional }\end{array}$ & $\begin{array}{l}\text { Provides higher data rates but is a } \\
\text { costly alternative }\end{array}$ \\
\hline
\end{tabular}

Some other types are also tailored in literature but are simply the derivatives of the mentioned types. Simply connecting a wired media between two devices are not enough for them to communicate. They should compromise on a common standard. This could be explained as a mail system is a communication media but both the sender and receiver should communicate in a common language. In our case the language means common voltage and current levels, timing sequences, appropriate delays and many more. Thus there comes a need to define a protocol for communication between the nodes. Some important and basic concepts are highlighted in section II. Along with them the bus attributes are also discussed. The requirements of the vehicular communication boosted the need for variety of protocols. Such requirements are enlisted in section III and the protocol architecture is discussed in section IV. The protocols which were separately identified earlier now very assumed to be a part of network and large number of networks was discovered thus the words networks and protocols were used interchangeably. The network topology used also had a significant effect on the performance of the network some basic topologies are also introduced in section $\mathrm{V}$. The work is concluded in section VI 


\section{BASIC CONCEPTS AND SYSTEM ATTRIBUTES}

A. Basic concepts:

1. Mastering: A bus connected with the number of nodes need a master to manage a bus. Initially only one node would act as master but as requirement for more control on bus rose the multi master concept was introduced. Now the control on bus became robust at the cost of increased complexity and higher cost. The congestion on the bus however could be catastrophic for Time critical applications.

2. Dominant \& Recessive State: When a bus is voltage is pulled high or low by an active switch element then the state of bus is said to be Dominant. Similarly for a Recessive state the bus voltage is passed through a passive element. Contentions are avoided by Dominant Over powering the Recessive. To make the concept of dominant and Recessive states crystal clear the figure 1 aids in the level of understanding.

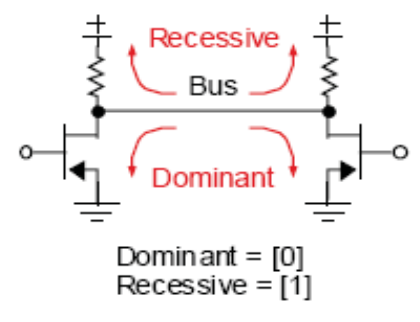

Figure 1: Dominant and Recessive states

3. Collision Handling: The collision occurring can be either handled by collision back off or by stoping all the nodes and retransmission. The former approach doesn't add any delay whereas the later introduces the delay and causes interruption. However the interruption time could be compensated by operating at higher data rates.

\section{$B$ System Attributes:}

The system attributes could be listed down as follows

- $\quad$ System cost

- Data Rates

- $\quad$ Physical layer requirements and control

- $\quad$ Through put

- $\quad$ Number of devices on the bus

- $\quad$ Error diagnosis

- $\quad$ Radiated EMI

System cost is the primary attribute because it is the major parameter upon usage of a bus or protocol. The communication bus became more famous by the name of the protocols used by them. In brisk it drives the marketability.

Different applications require different data rates we can classify as low, medium or high data rates. Earlier the data rate requirement was low and most of the vehicular machinery was mechanically controlled either by physical connections or by hydraulics. As the demand hiked for more advanced features there was considerable increase in the data rate. Bandwidth utilization became the new facet by the advent of infotainment used in vehicles. Thus the system requirements were now classified with respect to the domains.

\section{VEHICLE COMMUNICATION REQUIREMENTS}

Advances in the automotive electronics boosted the requirement list of the automotive communication. All the electronics in a vehicle is handled by a part name called Electronic Control Unit popularly known as ECU. Today's car had more than 3000 signals which are using more than 70 ECUs. The foetus approach was to add on an ECU for enhanced features but this approach failed as space and complexity exponentially increased. The following is a snapshot of requirement list for communication in automobile and is a demand driven and never ending paradigm.

The requirements highlighted by Nolte et al in [1] comprises of fault tolerance, determinism, bandwidth, flexibility and security. However the requirements drastically changed with the more usage of sensors and electronics. In order to keep the research live, a wide number of special interest groups emerged with the introduction of new protocols such as LIN consortia, CAN consortia, Flex ray etc. The automotive manufacturers came together and formed such groups some of these are Automotive Open System Architecture (AUTOSAR), , Society for Automotive Engineers SAE, JasPar, OPENSIG, AVnu alliance. The details of the protocols by each of these could be found on their web addresses mentioned in [5-10] 
An automobile system could be classified into eight typical subsystems as suggested by Nolte et al in [1] which are chassis, Air bag, Power train ,Body and Comfort, X by wire, Multimedia and infotainment, Wireless Telematics and Diagnostics. The requirement of each subsystem varies considerably. For some time the requirements were considered as per the subsystems but soon they were classified in different categories such as Control applications, Safety, Infotainment, Driver Assistance. For further exploration of each subsystem [1] and [2] are excellent sources.

The different domains necessitate different kinds of data rates thus Navet et al in [3] has described four classes and the domains which are tabled below

Table 2: Different domain requirements

\begin{tabular}{|l|l|l|}
\hline Class & Domain & $\begin{array}{l}\text { Protocol } \\
\text { examples }\end{array}$ \\
\hline A & Body & LIN \\
\hline B & $\begin{array}{l}\text { Communication } \\
\text { between ECUs }\end{array}$ & $\begin{array}{l}\text { J1850 and } \\
\text { low speed } \\
\text { CAN }\end{array}$ \\
\hline C & $\begin{array}{l}\text { Powertrain and } \\
\text { chassis }\end{array}$ & $\begin{array}{l}\text { High speed } \\
\text { CAN }\end{array}$ \\
\hline D & Multimedia data & MOST,LVDS \\
\hline
\end{tabular}

\section{AUTOMOTIVE PROTOCOL ARCHITECTURE}

The communication system in a vehicle is similar to the OSI layered architecture. The OSI consists of seven layers which are Physical ,Data link, Network, Transport, Session, Presentation and Application Layer whereas the automobile architecture had similar functions just some functions are carried out in a single layer. The both can be illustrated in figure 2 .
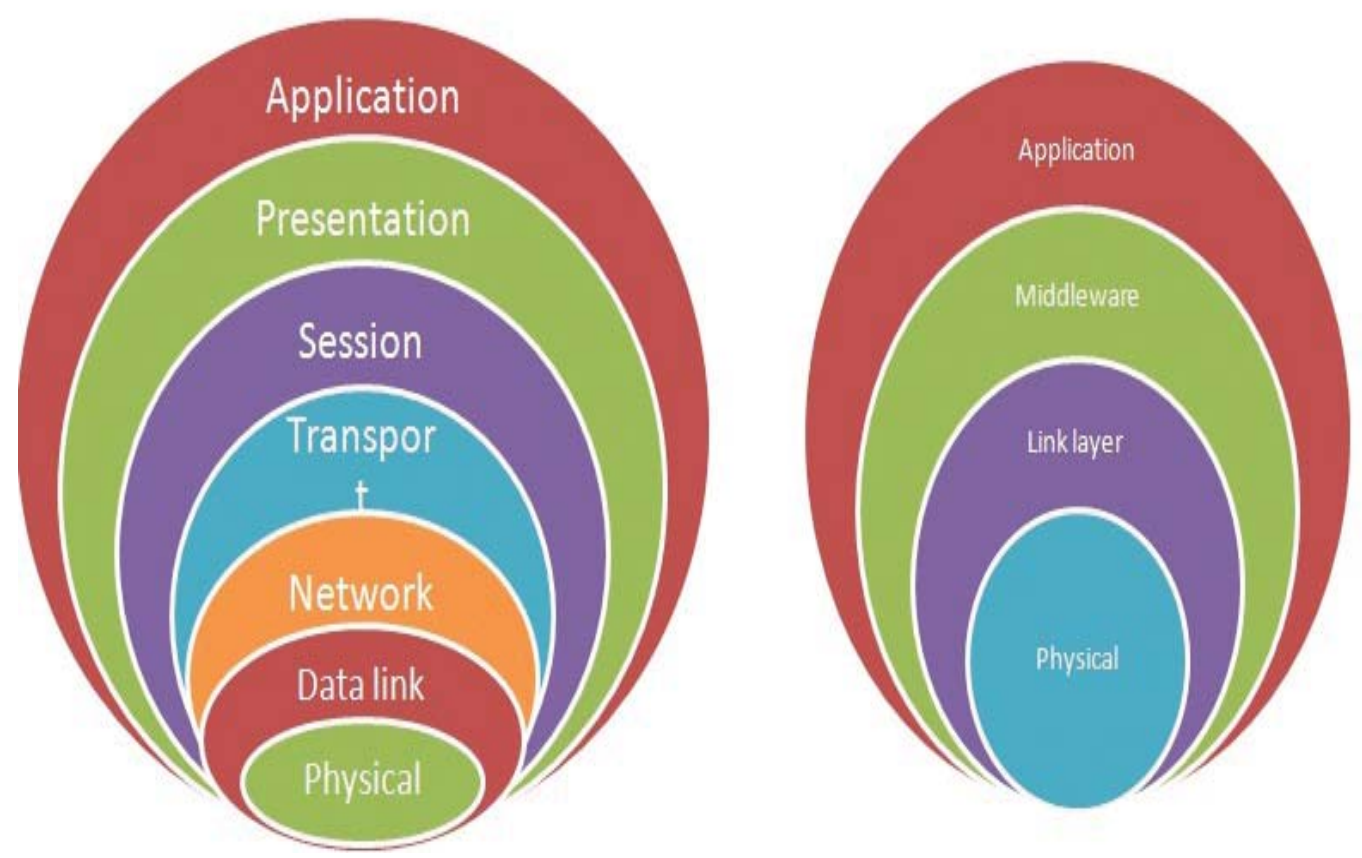

Figure 2: Seven OSI layers and Four Automobile Communication layers 
As time passed the protocol names were not to popular instead the networks became very shiny and the network embodied the protocols and other allied requirements. The protocol at different layers exhibit different features and are applied for different applications. Each layer has a number of protocols researched out over the years and are tabled below

Table 3: Different layers and Protocols

\begin{tabular}{|l|l|}
\hline Layer & Protocols \\
\hline Physical Layer & $\begin{array}{l}\text { LIN,CAN, Flex } \\
\text { ray,MOST and LVDS }\end{array}$ \\
\hline Link Layer & $\begin{array}{l}\text { IEEE802.1Q, AVB } \\
\text { Ethernet and } \\
\text { TTEthernet }\end{array}$ \\
\hline Middleware & AUTOSAR \\
\hline Application & Next Gen Ethernet \\
\hline
\end{tabular}

In order to introduce all the protocols only its progress is summarized and a very precise description is exhibited. Local Interconnect network was found in late 1990's by a group of five automotive giants. The first version which was fully implemented was LIN 1.3 in 2002. In the next year LIN 2.0 was introduced with additional diagnostic features. LIN had no bus arbitration as it was a single master network wherein up to sixteen nodes could connect. Among them one would act as master and others as slave. LIN can be applied in automotive at the roof, steering wheel, seat, engine, door and window. It provides good alternative where bandwidth and speed are not point of consideration. Now the LIN consortium is handed over to the ISO and is numbered ISO 17987. The latest version of LIN is $2.2 \mathrm{~A}$.

Controller Area Network is another protocol introduced in 1986 at the SAE conference in Michigan by Robert Bosch. Later it was made market ready by Phillips and Intel. In 1991 Bosch introduced CAN 2.0 with two magnitudes of identifiers one with 11 and other 29 bit identifier. Both were named 2.0A and B respectively. ISO released the 11898 with two variants 1 consists of data link layer and 2 with physical layer for good speed CAN. To cut down cost it also introduced 11898-3 for low speed CAN. Soon CAN 2.0 versions were superseded by the ISO versions. Later late in 2012 the CanFD was introduced which exhibited Flexible Data rates. The utility of CAN is very high thus today currently the on board Diagnostics version II also uses CAN and other four protocols. The On board diagnostics is now popularly known as OBD II and is made mandatory for most kinds of European vehicles.

CAN is a multi master protocol wherein it allows more than one master on the bus. It has the non destruction bus arbitration feature which avoids delay generally caused during retransmissions. It had good error analysis features and became very popular in the European countries. It found its application in variety of fields. In industries it is used as a field bus. Some of the applications are Anti lock Braking System, Electronic Power Steering, Adaptive Cruise Control etc. Device net which is more popular in automation and embedded industries is completed having the CAN framework and is a strong derivative of CAN. However CAN is an event triggered network and bus traffic becomes uncertain. In case of safety critical applications the CAN was not able to deliver much due to its event based nature. Some efforts are still going on to make it time triggered harness it as low cost substitute and provide advanced features. 


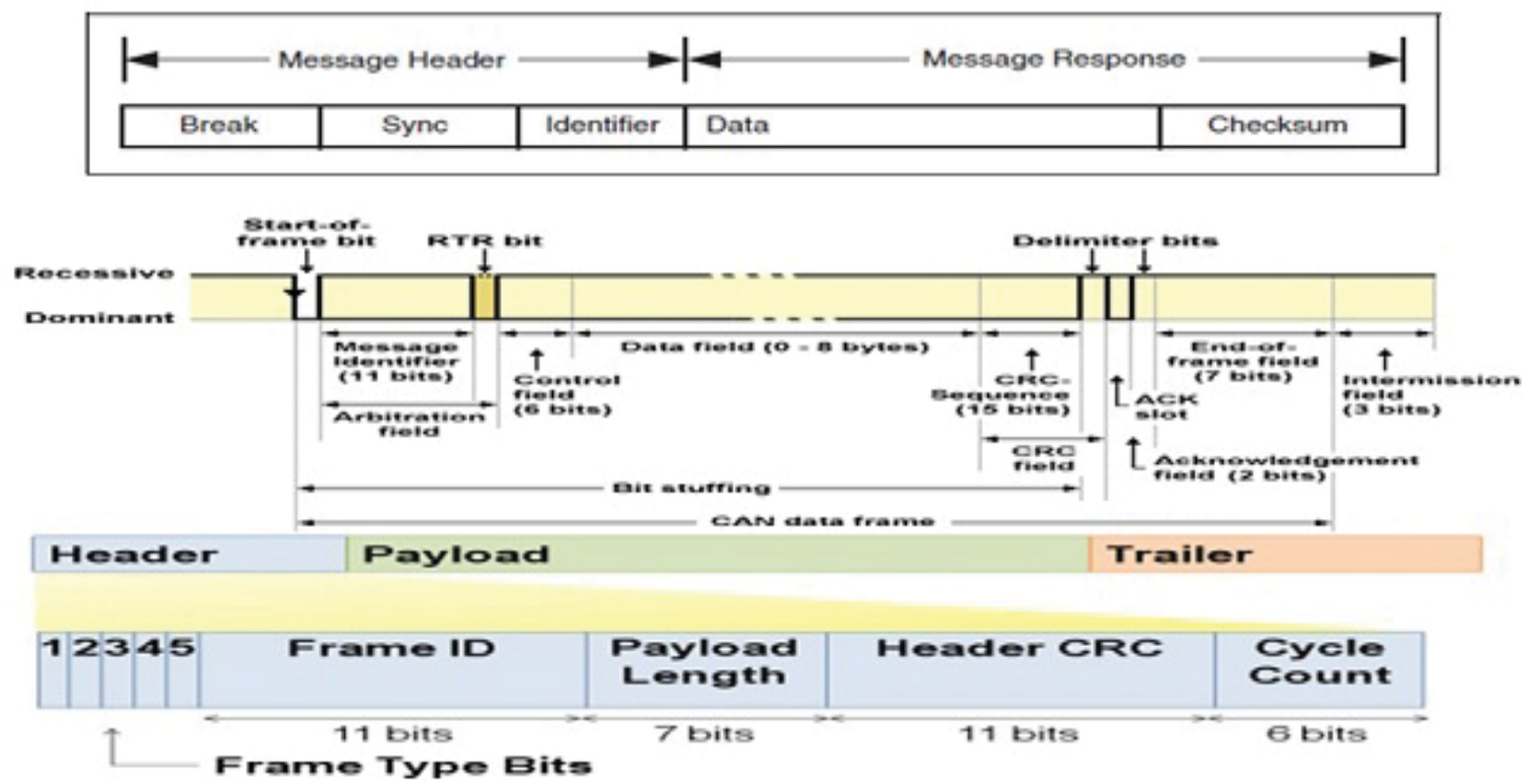

Figure 3: LIN, CAN and Flex ray frame formats (top to bottom i.e. first LIN and last Flex ray)

BMW, Bosch, Daimler Chrysler, GM, Motorola, Philips and Volkswagen formed a consortium and resulted in Flex ray protocol. It illustrates both the time triggered and event triggered nature. Time triggered nature enables to make the traffic on the bus predictable. In other works everything is planned before hand whereas in event triggering only and only on occurrence of an event the task communication starts. The Flex ray comprised of two windows one which exhibiting time and event triggering. The event triggered window used the flexible TDMA protocol for communication whereas the time triggered window uses the TDMA MAC protocol. The figure 4 depicts the three frame formats of LIN, CAN and Flex ray respectively going from top to bottom.

Media Oriented System Transport was targeted on the multimedia data networking. Much of the details of these protocols are secured by the manufactures thus cannot be highlighted. It offers a data rate of $150 \mathrm{mbps}$ and much more belongs to the infotainment domain or subsystem.

Low Voltage Differential Signalling is basically under the non automotive specific standard and much more useful in audio and video communication. With the increasing integration and usage of audio and video parameters in driver assistance systems, the LVDS becomes important for automotives. It uses a twisted pair cables for transmission and offers a very high data transfer rates of up to $655 \mathrm{mbps}$.

The current research is going on the time triggered and event triggered nature of protocols. Considering both on the similar grounds the former is very useful in time critical scenario. The hard real time requirements are mandatory which imparts the communication media traffic to be predictable. The later causes a burst of data to be communicated with the occurrence of the events. Larger the number of events greater would be the changes for congestion or sometimes failure. The event triggered is suitable for soft real time applications where missing the deadline does not create any catastrophic situation.

\section{NETWORK TOPOLOGIES}

Network topologies play crucial role in deciding the performance of any network. It talks about the pattern in which the nodes are connected in a network. The basic types of it are bus, star, ring, mesh, tree, hybrid, daisy chain, double star etc. The researchers are actively engaged in the exploration of new network topologies for enhanced performance. The following figure depicts the different types of network topologies used. 


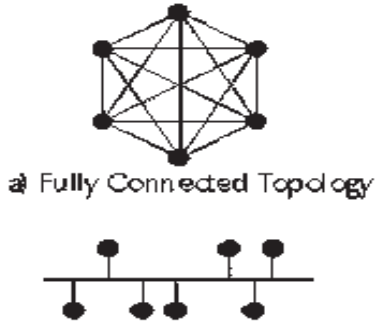

bi Bus Topology

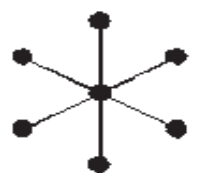

d Star Topdogy

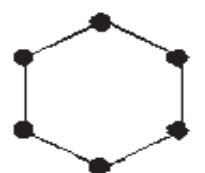

d) Ring Topdogr


Nodes Eranches

Figure 4: Different network topologies

The effect of different networking topologies however is out of the scope of this paper but interested readers could find it in [4].

\section{CONCLUSION}

Looking at the growth of the automotive electronics, in the late 1990's the Local Interconnect network was very famous for low data rate applications. CAN emerged very strongly over the LIN protocol but could not totally replace CAN because of its inexpensiveness. CAN introduced in late 90's had a variety of versions such as SWCAN, high speed CAN, TTCAN ,CAN kingdom, Byte flight ,CANOpen, CANFD and many more. As the demand hiked the safety and security aspects of the automotive gained attention and there was a need of time triggered protocols for safety critical applications. The deliberate effort was made by researchers to mould CAN into a time triggered one but cost was the main hurdle. Flex ray provided both the event and time triggered window which full filled the need of both the hard real time and soft real time applications. Flex ray also cut down the upgradation time requirements as compared to CAN. With reference to discussions in literatures CAN firmware upgrade takes hours whereas the Flex ray completes in few minutes. Network topologies also are an important aspect needs to be taken into consideration. Ethernet is predicted to become backbone of the automotive electronics but alas the shift from the current technologies to the next generation Ethernet based one would be very costly thus the integration of current and IP based technologies could provide a cost effective solution.

\section{ACKNOWLEDGEMENT}

I would like to acknowledge all my well wishers who stood by my side and supported my sincere efforts. I would like to thank all my friends and family who were constantly my source of inspiration and always supported me 


\section{REFERENCES}

[1] Nolte et al , "Automotive Communication: Past Present and Future", Proceedings of $10^{\text {th }}$ IEEE Conference ETFA, vol 1 p 9922005

[2] Shane Touhy et al , "Intra Vehicle Networks : A Review ", IEEE transactions on Intelligent Transportation System vol 16 NO 2 2015

[3] Navet et al , “ Trends in Automotive Communications” ,Proceedings of the IEEE vol 93 ,NO 6 pp 1204-1223 2005

[4] Rahmani et al ,"Performance Analysis of Different network topologies for In Vehicle audio and Video Communication", $2^{\text {nd }}$ Proceedings IEEE 2008

[5] OPEN Alliance SIG,OPEN Alliance: About 2012

[6] AVnu Alliance www.avnu.org

[7] JasPar 2013 www.jaspar.jp/english

[8] AUTOSAR www.autosar.org

[9] CAN www.canopen.org

[10] LIN , LIN specification package 2.0 